\title{
Defect Tolerance for Gracefully-Degradable Microfluidics-Based Biochips*
}

\author{
Fei Su and Krishnendu Chakrabarty \\ Department of Electrical \& Computer Engineering \\ Duke University, Durham, NC 27708 \\ E-mail: \{fs, krish\}@ee.duke.edu
}

\begin{abstract}
Defect tolerance is an important design consideration for microfluidics-based biochips that are used for safety-critical applications. We propose a defect tolerance methodology based on graceful degradation and dynamic reconfiguration. We first introduce a tile-based biochip architecture, which is scalable for large-scale bioassays. A clustered defect model is used to evaluate the graceful degradation method for tile-based biochips. The proposed schemes ensure that the bioassays mapped to a droplet-based microfluidic array during design can be executed on a defective biochip through operation rescheduling and/or resource rebinding. Real-life biochemical procedures, namely polymerase chain reaction (PCR) and multiplexed in-vitro diagnostics on human physiological fluids, are used to evaluate the proposed defect tolerance schemes.
\end{abstract}

\section{Introduction}

Microfluidics-based biochips have seen considerable growth over the past decade [1]. Such composite microsystems, also known as bio-MEMS or lab-on-a-chip, offer a number of important advantages over conventional laboratory methods; these include design flexibility, smaller size, lower cost, and higher sensitivity. Biochips enable the control of small amounts (e.g., nanoliters) of fluids, thus reducing sample size, reagent volume, and power consumption. In recent years, microfluidicsbased biochips have been developed for enzymatic analysis (e.g., glucose and lactate assays), DNA analysis (e.g., PCR and nucleic acid sequence analysis), proteomic analysis involving proteins and peptides, immuno-assays, and toxicity monitoring $[1,2]$.

Most microfluidics-based biochips of today consist of microchannels, microvalves, and micropumps that are permanently etched in glass, plastic or silicon substrates, and their operation is based on the principle of continuous flow [1]. However, they are neither scalable nor reconfigurable for largescale or concurrent bioassays. A promising alternative is to manipulate liquids as discrete microdroplets using the principle of electrowetting. This novel droplet-based microfluidics technique is often referred to in the literature as "digital microfluidics" [3]. In comparison to continuous-flow systems, they are easier to manufacture, and they facilitate dynamic reconfigurability as well as a scalable system architecture [3]. By using a two-dimensional array of control electrodes, microdroplets can be easily moved to any arbitrary location in this array; thereby many common microfluidic operations for bioassays can be easily performed.

An emerging application area for digital microfluidics is clinical diagnostics. The in-vitro measurement of glucose and other metabolites, such as lactate, glutamate and pyruvate, in human physiological fluids is important for the clinical diagnostics of metabolic disorders [2]. The feasibility of performing a colorimetric enzyme-kinetic assay (e.g., glucose assay or lactate assay) on a digital microfluidics-based biochip has recently been demonstrated experimentally [4]. This technology is expected to develop rapidly to facilitate

* This research was supported by the National Science Foundation under grant number IIS-0312352. immediate point-of-care diagnosis of diseases. Digital microfluidics-based biochips are also capable of continuous sampling and real-time testing of air/water samples for biochemical toxins and other pathogens; therefore they can serve as an always-on "bio-smoke alarm" to offer an early warning capability to citizens.

The 2003 International Technology Roadmap for Semiconductors (ITRS) anticipates that microfluidic biochips will soon be integrated with electronic components in systemon-chip (SOC) design; this will constitute one of the major system-level design challenges that will be faced beyond 2009, when feature sizes shrink below $50 \mathrm{~nm}$ [5]. This document also recognizes the need for new test methods for disruptive device technologies that underly microfluidics, and highlights it as one of the five difficult test challenges beyond 2009. Advances in fabrication technology and the increased integration levels of digital microfluidics-based biochips will allow the simultaneous execution of many bioassays in a single microfluidic array. However, shrinking processes, new materials, and the underlying multiple energy domains will make digital microfluidics-based biochips susceptible to manufacturing defects. Moreover, some manufacturing defects are expected to be latent and they may manifest themselves during field operation of the biochips. Since many microfluidics-based biochips are intended for safety-critical applications, system dependability is an essential performance parameter. Therefore, defect tolerance is an important design consideration for digital biochips that are targeted towards biomolecular recognition and clinical diagnostics applications.

Defect tolerance can be achieved by including redundant elements in the system; these elements can be used to replace faulty elements through reconfiguration techniques. An alternative method is based on graceful degradation, in which all elements in the system are treated in a uniform manner, and no element is designated as a spare. In the presence of defects, a subsystem with no faulty element is first determined from the faulty system. This subsystem provides the desired functionality, but with a gracefully-degraded level of performance (e.g., longer execution time). Due to the dynamic reconfigurability of digital microfluidics-based biochips, the microfluidic components (e.g., mixers) used during the bioassay can be viewed as reconfigurable virtual devices. For example, a $2 \times 4$-array mixer (implemented using a rectangular array of control electrodes-two in the X-direction and four in $\mathrm{Y}$ direction) can easily be reconfigured to a $2 \times 3$-array mixer or a $2 \times 2$-array mixer. This feature facilitates the use of graceful degradation to achieve defect tolerance in digital biochips.

In this paper, we propose a defect tolerance methodology based on graceful degradation and dynamic reconfiguration. We first introduce a novel tile-based architecture, which is scalable to large bioassay applications. We next introduce a clustered defect model, which is used to evaluate the graceful degradation approach for a tile-based digital biochip. The goal of reconfiguration is to ensure that the bioassays mapped to the 
microfluidic array during design can be executed on the defective biochip through operation rescheduling and/or resource rebinding. Two examples of real-life biochemical procedures, namely PCR and multiplexed in-vitro diagnostics on human physiological fluids, are used to evaluate the proposed defect tolerance scheme. Simulation results show that the tile-based design increases the yield of digital biochips, i.e., the array can be reconfigured and a set of bioassays can be successfully remapped to execute on a faulty array.

The organization of the remainder of the paper is as follows. In Section 2, we discuss related prior work. Section 3 presents an overview of digital microfluidics-based biochips. It also introduces a scalable tile-based architecture of a microfluidic array. Section 4 discusses manufacturing defects and proposes a clustered defect model based on a spatial defect distribution. In Section 5, we study the graceful degradation of a tile of biochips in the presence of a defect cluster. Different reconfiguration schemes based on operation rescheduling and/or resource rebinding are also presented. In Section 6, PCR and multiplexed in-vitro diagnostics on human physiological fluids are used to evaluate the proposed defect tolerance scheme. Finally, conclusions are drawn in Section 7.

\section{Related Prior Work}

Defect tolerance techniques for integrated circuits (ICs) are well known [6, 7]. Defect tolerance methods based on reconfiguration were initially used for memory chips [7]; they are now also used for various array-based architectures such as processor arrays (PAs) or FPGAs [8]. Due to the reconfigurability inherent in PAs and FPGAs, graceful degradation approach can also be used for defect tolerance in these systems [9].

Microelectromechanical systems (MEMS) is a relatively young field compared to ICs. Recently, testing techniques including fault simulation and fault modeling for surface micromachined MEMS have received attention [10, 11, 12]. Attempts have also been made to make MEMS defect-tolerant. For example, design-for-manufacturing (DFM) and design-fortestability (DFT) methodologies have been incorporated in the design process for MEMS [13].

Microfluidics differs from MEMS in the underlying energy domains and in its working principles. Hence, defect tolerance techniques for MEMS cannot be directly applied to microfluidic biochips. Recently, fault modeling, fault simulation, and a DFT methodology for continuous-flow microfluidic systems have been proposed [14, 15]. For digital microfluidics-based biochips, research to date has focused on fault classification and a unified test methodology. Faults are classified as either manufacturing or operational, and techniques have been developed to detect these faults by electrostatically controlling and tracking droplet motion [16]. This cost-effective test methodology facilitates concurrent testing for digital microfluidics-based biochips, which allows fault testing and biomedical assays to run simultaneously on a microfluidic biochip. Test planning and test resource optimization are motivated by the need for concurrent testing [17]. A system design methodology has been proposed to apply classical synthesis techniques to biochip design [18]. These CAD techniques facilitate the incorporation of defect tolerance in the design of microfluidics-based biochips.

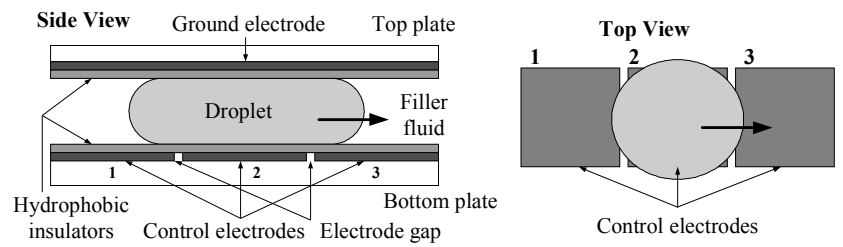

Figure 1: Basic cell used in a digital microfluidics-based biochip.

\section{Tile-Based Architecture}

The basic cell of a digital biochip consists of two parallel glass plates between which droplets containing liquid samples are sandwiched, as shown in Figure 1. The bottom plate contains a patterned array of independent control electrodes ( $200 \mathrm{~nm}$ thick layer of chrome), and the top plate contains a ground electrode formed by a layer of transparent indium tin oxide (ITO). Droplets are surrounded by a filler medium such as $1 \mathrm{cSt}$ silicone oil to prevent evaporation and to reduce the droplet actuation voltage. In addition, Parylene C $(\sim 800 \mathrm{~nm})$ is used to insulate droplets from the electrode array, and a thin layer of hydrophobic Teflon AF $1600(\sim 50 \mathrm{~nm})$ is coated on the top and bottom plates to decrease the wettability of the surface.

Droplets are actuated through electrowetting wherein the interfacial tension of the droplet is modulated with an electric field. A control voltage is applied to an electrode adjacent to the droplet (e.g., electrode 3 in Figure 1) and at the same time the electrode just under the droplet (e.g., electrode 2 in Figure 1) is deactivated. Thus, the charge in the droplet/insulator interface that is accumulated over the activated electrode results in an interfacial tension gradient, which consequently causes the transportation of the droplet. By varying the electrical potential along a linear array of electrodes, nanoliter-volume droplets can be transported along this line of electrodes. The velocity of the droplet can be controlled by adjusting the control voltage $(0 \sim 90 \mathrm{~V})$, and droplets have been observed to move with velocities up to $20 \mathrm{~cm} / \mathrm{s} \mathrm{[3].} \mathrm{Furthermore,} \mathrm{based} \mathrm{on} \mathrm{this}$ principle, droplets can be transported freely to any location on a two-dimensional array without the need for pumps and valves.

Using a two-dimensional array, many common operations for different bioassays can be performed, such as sample movement (transport), temporary sample preservation (store), and the mixing of different samples (mix). For instance, the store operation is performed by applying an insulating voltage around the droplet. The mix operation is used to route two droplets to the same location and then turn them around some pivot points. Note that these operations can be performed anywhere on the array, whereas in continuous-flow systems they must operate in a specific micromixer or microchamber. This property is referred to as the reconfigurability of a digital biochip. The configurations of the microfluidic array, i.e., the routes that droplet travel and the rendezvous points of droplets, are programmed into a microcontroller that controls the voltages of electrodes in the array.

We next describe a tile-based architecture that is especially suitable for the execution of a set of bioassays on an array with defect sites. This regular architecture consists of an array of reconfigurable tiles, each of which has the same original configuration and can be used to perform the basic common microfluidic operations of bioassays, e.g. transport, mix, or detection. An example of the tile structure is shown in Figure 2, where a $2 \times 4$-array mixer is located in the center of the tile, and it can be used for rapid droplet mixing. In addition, there are 


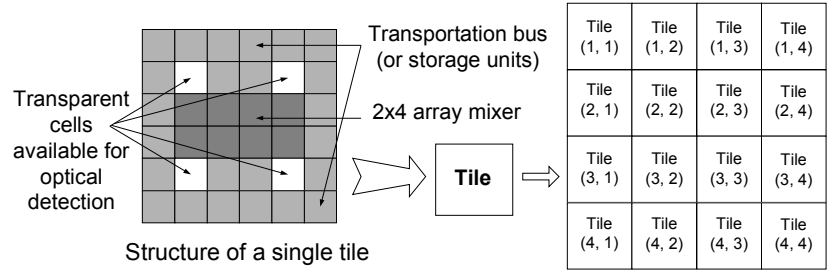

Figure 2: Tile-based architecture of microfluidic biochips.

four transparent cells that serve as feasible locations for optical detectors. Optical detection is commonly used to quantitatively measure the result of bioassays; it determines the concentration of the biochemical sample (end point of a reaction) by observing the change in its absorbance properties. To perform optical detection, the optical detector consisting of a LED and a photodiode can be added to the location of the transparent cell of a tile, where the droplet containing the bioassay product can be detected. The boundary cells in a tile can serve as transportation bus or storage unit for droplets. Thus, the basic microfluidic operations can be performed in a single tile. Moreover, since each microfluidic cell in a tile has an independent control electrode, the reconfiguration of a tile can be easily carried out to increase yield.

\section{Physical Defects and a Clustered Defect Model}

Digital microfluidics-based biochips are fabricated using standard microfabrication techniques; details of the fabrication process are described in [19]. Due to the underlying mixed technology and multiple energy domains, microfluidic biochips exhibit unique failure mechanisms and defects. Faults may be caused by the following manufacturing defects [16]:

- Dielectric breakdown: The breakdown of the dielectric at high voltage levels creates a short between the droplet and the electrode. When this happens, the droplet undergoes electrolysis, thereby preventing further transportation.

- Short between adjacent electrodes: If a short occurs between two adjacent electrodes, the two electrodes shorted effectively form one longer electrode. When a droplet resides on this electrode, it cannot overlap its adjacent electrodes. As a result, the actuation of the droplet can no longer be achieved.

- Open in the metal connection between the electrode and the control source: This defect results in a failure in activating the electrode for transport.

Manufacturing defects can also cause geometrical parameter deviations. The deviation in insulator thickness, electrode length, and height between parallel plates may exceed their tolerance values during fabrication. In addition, some imperfections in the chrome layer (i.e., control electrode) during manufacturing might have a negligible effect initially after production. However, these imperfections may cause wear-andtear during the operation of the microfluidic biochip, consequently leading to the degradation of the electrode. An image of such a faulty electrode, photographed in our laboratory for a manufactured array, is shown in Figure 3 [19].

Based on the unified detection mechanism proposed in [16], stimuli droplets containing a conductive fluid (e.g., KCL solution) from the droplet source are transported through the array (i.e., traversing the cells) following the designed test plan to detect the faulty cells. After faulty cells are identified in the array, reconfiguration techniques can be used to bypass these faulty cells to tolerate defects.

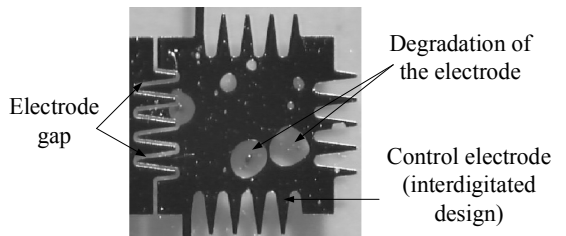

Figure 3: Top view of a faulty cell: electrode degradation.

To develop an efficient defect tolerance and reconfiguration scheme, a realistic defect model for microfluidic biochips must be introduced. As in the case of VLSI circuits [20,21], defects in microfluidic biochips tend to occur in clusters due to imperfections that span multiple neighboring cells in the array. For example, shorts between adjacent electrodes caused by a spot defect (e.g., undesirable chemical and airborne particles) usually result in multiple neighboring faulty cells. This physical phenomenon is referred to as defect clustering.

We attempt to deal with defect clustering by introducing a defect model that statistically describes the spatial distribution of defects in a tile-based microfluidic array. In this model, we assume that each tile contains one defect cluster with probability $q$ independent of the other tiles. Let $p=1-q$ denote the probability that a single tile is defect-free. We refer to $p$ as the survival probability of a single tile. The defect cluster affects a certain region of a faulty tile, which is referred to as the influence region of this defect cluster. Each microfluidic cell that is in the influence region of a defect cluster is rendered faulty. In the proposed model, the influence region of a defect cluster is a square and it can be located anywhere in a faulty tile; its size is uniformly distributed from $1 \times 1$ (a single faulty cell) to $m \times m$ (multiple faulty cells), where $m$ is less than the number of rows/columns in a tile. The assumptions of arbitrary defect cluster location and random cluster size complicate the graceful degradation and reconfiguration technique, but they make the defect model more realistic.

\section{Graceful Degradation and Reconfiguration}

For the clustered defect model described in Section 4, we next investigate a graceful degradation approach that retains full or partial functionality of a faulty tile. We also develop reconfiguration schemes to reallocate the operations of an assay performed in a gracefully-degradable tile-based platform.

Many common microfluidic operations can be implemented using different droplet-based components that offer different performance levels. For example, the mixing of samples and reagents is a critical microfluidic operation for bioassays. Recent experiments have shown that mixing can be performed in different types of droplet mixers, such as $2 \times 4$-array mixers, $2 \times 3$-array mixers, $2 \times 2$-array mixers, or 4 -electrode linear array mixers [22]. The mixing times for various mixers are listed in Table 1. Note that in these experiments, every mixer was assumed to have the same geometric parameters, i.e., an electrode pitch of $1.5 \mathrm{~mm}$ and the gap height between the two plates of $600 \mu \mathrm{m}$. Sample droplets contained $1 \mathrm{mM}$ fluorescein, $0.125 \mathrm{M} \mathrm{KCL}$ and $0.125 \mathrm{M} \mathrm{NaOH}$, with viscosity of $1.397 \mathrm{cP}$, while reagent droplets contained $0.125 \mathrm{M} \mathrm{KCL}$ and $0.125 \mathrm{M}$ $\mathrm{NaOH}$, with viscosity of $1.373 \mathrm{cP}$. The actuation voltage was set to $50 \mathrm{~V}$ in experiments. The fastest mixing time for two 1.4 $\mu 1$ droplets was 2.9 seconds using a $2 \times 4$-array mixer operating at $16 \mathrm{~Hz}$. On the other hand, the mixing time using a $2 \times 2$-array mixer was as high as 9.95 seconds, while it required much $50 \%$ microfluidic cells compared to the $2 \times 4$ array mixer. 
Table 1: Mixing times for various types of mixers [22].

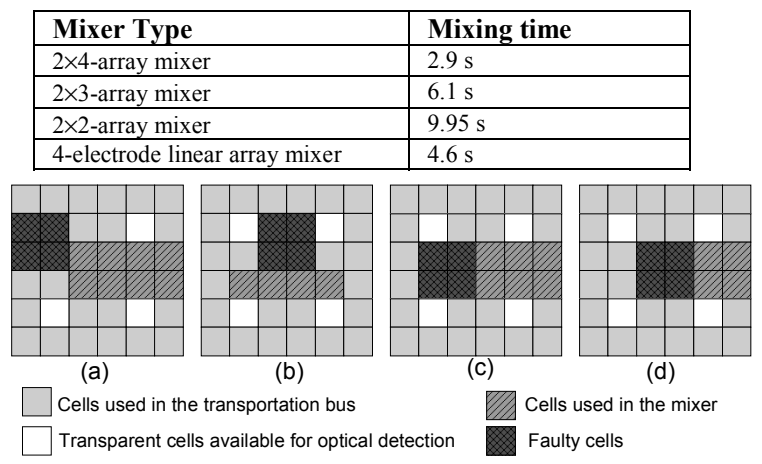

Figure 4: (a) Faulty tile with a reconfigured $2 \times 4$-array mixer, (b) Faulty tile with a reconfigured 4-electrode linear array mixer, (c) Faulty tile with a reconfigured $2 \times 3$ array mixer, (d) Faulty tile with a reconfigured $2 \times 2$-array mixer.

Table 2: Probability of reconfiguring a $2 \times 4$ array (denoted by $P_{r}$ ).

\begin{tabular}{|c|c|c|c|c|c|}
\hline$P_{r}$ Defect cluster size & $1 \times 1$ & $2 \times 2$ & $3 \times 3$ & $4 \times 4$ & $5 \times 5$ \\
\hline $2 \times 4$-array mixer & 0.89 & 0.64 & 0 & 0 & 0 \\
\hline 4-electrode linear array mixer & 0.11 & 0.24 & 0.5 & 0 & 0 \\
\hline $2 \times 3$-array mixer & 0 & 0.08 & 0.25 & 0 & 0 \\
\hline $2 \times 2$-array mixer & 0 & 0.04 & 0.25 & 0.67 & 0 \\
\hline Reconfiguration not possible & 0 & 0 & 0 & 0.33 & 1 \\
\hline
\end{tabular}

Due to the reconfigurability of the microfluidic array, a mixer can be easily reconfigured to other configurations by changing the control voltages for the corresponding electrodes. Consider a tile with a $6 \times 6$ array as described in Section 3. When a defect cluster occurs in this tile causing some cells of the mixer to be faulty, the $2 \times 4$-array mixer in the original configuration can be reconfigured to a $2 \times 3$-array mixer, or a $2 \times 2$-array mixer, or a 4-electrode linear array mixer to avoid faulty cells. With this approach, the mix operation of bioassays can still be carried out in this faulty tile, but with degraded performance, i.e., increased mixing time. Some examples of reconfiguration corresponding to a $2 \times 2$ defect cluster are shown in Figure 4. In order to minimize the performance degradation, we first attempt to find a $2 \times 4$-array configuration for the mixer through appropriate reconfiguration. If this attempt fails, a feasible configuration is chosen from the alternatives of a 4electrode linear array mixer, a $2 \times 3$-array mixer, or a $2 \times 2$-array mixer, and these alternatives are ranked based on their performance.

Recall that in the proposed defect model, a defect cluster with a random size of the influence region is assumed to be arbitrarily located in the faulty tile. Table 2 lists the probability that the $2 \times 4$-array mixer in a tile can be reconfigured as the mixer indicated by the row label (except the last row, which indicates the probability that graceful degradation via reconfiguration is not possible). Every column in the table adds up to 1 . For example, if a defect cluster in the faulty tile has the influence region of $1 \times 1$ size (i.e., covering one single cell), there are a total of 36 different cases for the possible location of this defect cluster. Among them, only four cases (when the defect cluster is located near to the center of the tile) need the reconfiguration of the mixer from the original configuration to the 4 -electrode linear array. For the other cases, the $2 \times 4$-array configuration for the mixer can be maintained. Therefore, as indicated in the first column in the table, the probability that the $2 \times 4$-array mixer in this faulty tile can be reconfigured as a 4 electrode linear array mixer is 0.11 , and the probability that the $2 \times 4$ array mixer can still be used is 0.89 .

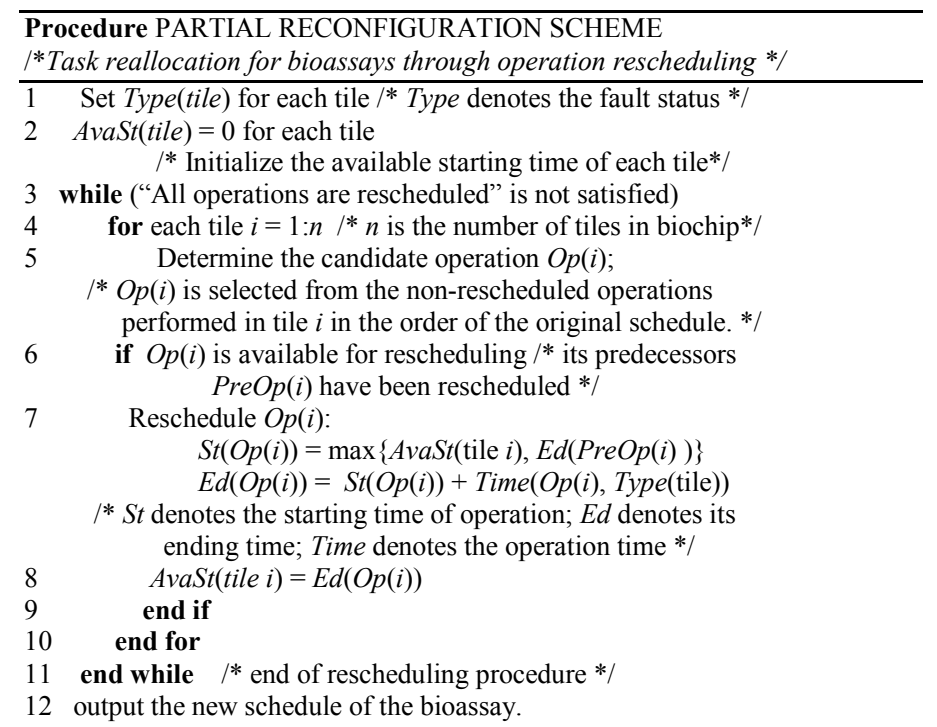

Figure 5: Pseudocode for the partial reconfiguration scheme.

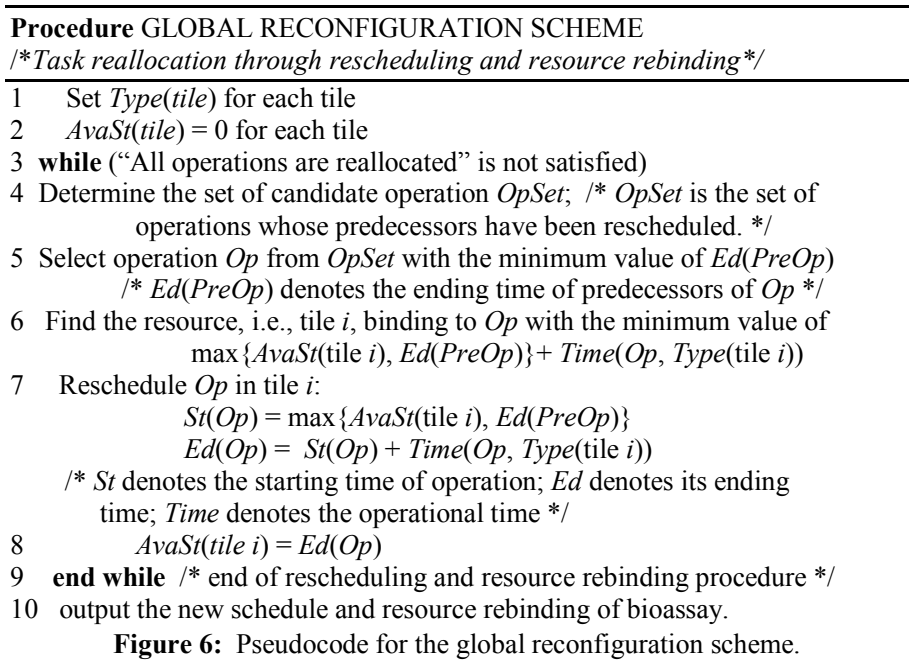

Based on the proposed graceful degradation method applied to a single tile, a higher-level scheme is needed to efficiently reconfigure and reallocate the operations of a bioassay that has been mapped to a tile-based microfluidic biochip. Two different reconfiguration schemes are proposed in this paper. The first scheme is based on operation rescheduling. Only the operations that are bound to faulty tiles and their successors need to be rescheduled; the others are left unchanged. The pseudocode for this scheme is shown in Figure 5. Since not all the operations of an assay need to be rescheduled, this scheme is referred to as partial reconfiguration. On the other hand, the second method uses not only operation rescheduling but also resource rebinding. In this scheme, architectural-level synthesis is used to provide a new mapping of assay operations to time-steps, as well as a new assignment of assay operations to biochip resources. The goal of the synthesis procedure is to minimize the assay completion time, and thereby the performance degradation. Architectural-level synthesis for microfluidicsbased biochips is NP-complete [18]. Therefore, a simple but efficient heuristic algorithm is used in the reconfiguration scheme; its pseudocode is shown in Figure 6. This scheme may affect an operation of an assay that has been bound to a faultfree tile. Therefore, it is referred to as global reconfiguration. While partial reconfiguration is computationally simpler, global reconfiguration based on an architectural-level synthesis 
procedure yields lower completion time for a set of bioassays, thereby leading to less performance degradation.

\section{Simulation Results}

Two real-life biochemical applications, namely PCR and in-vitro diagnostics on human physiological fluids, are used to evaluate the proposed graceful degradation and reconfiguration schemes. The feasibility of performing these bioassays on a digital microfluidics-based biochip has recently been demonstrated experimentally $[4,23]$.

We perform Monte-Carlo simulation to evaluate the proposed schemes. During each run of the simulation, the cells in the tile-based microfluidic array are randomly chosen to fail with a probability defined by the clustered defect model. We then attempt to tolerate these defects through graceful degradation. The operations of a complete bioassay procedure are reallocated to ensure its functionality via partial reconfiguration and global reconfiguration, respectively. To evaluate the effectiveness of graceful degradation, we consider a baseline scheme that attempts to carry out reconfiguration without graceful degradation. In this baseline scheme, we attempt to retain the original configuration (e.g., $2 \times 4$ array) for a microfluidic component (e.g., mixer) via reconfiguration when some cells are deemed faulty. If the original configuration cannot be maintained, this component is considered unrepairable. If some bioassay operation is scheduled in an unrepairable component, the microfluidic biochip containing this component cannot be made defect-tolerant.

The effectiveness of various reconfiguration schemes can be determined using two metrics. The first metric is the yield $\mathrm{Y}$, which is defined here as the percentage of defect-tolerant biochips for the given clustered defect model. The yield is averaged over a large number (e.g., 10000) of simulation runs, when faults are injected based on the clustered defect model in each run. A goal of the proposed graceful degradation and reconfiguration schemes is to increase the yield. The second metric, referred to as degradation level DEGR, measures the percentage increase in the bioassay processing time after reallocation. Let $T_{1}$ be the bioassay processing time for a defect-free microfluidic array. Let $T_{2}$ be the processing time after graceful degradation. The metric DEGR is defined as: DEGR $=\left(\left(T_{1}-T_{2}\right) / T_{2}\right) \times 100 \%$. As in the case of metric $Y$, the value of $T_{2}$ is averaged over a large number of simulation runs, where faults are injected in each run using the clustered defect model. This metric quantifies the increase in processing time for the graceful degradation approach. Clearly, for the simple reconfiguration scheme without graceful degradation, this metric is always 0 . In addition, we also compare the computation cost for different reconfiguration schemes.

\subsection{Evaluation example 1: PCR}

PCR is one of the most common techniques for DNA analysis [24]. It is used for rapid enzymatic amplification of specific DNA fragments. PCR can amplify genomic DNA exponentially using temperature cycles. Here, we use the mixing stage of PCR as an example to evaluate the proposed defect tolerance schemes. The assay protocol of PCR can be modeled by a sequencing graph [24], as shown in Figure 7, where $M_{i}$ denotes the mixing operation.

A tile-based microfluidic biochip with $2 \times 2$ tiles is used to implement the PCR mixing stage, as shown in Figure 8(a). An original schedule for the bioassay operations and resource

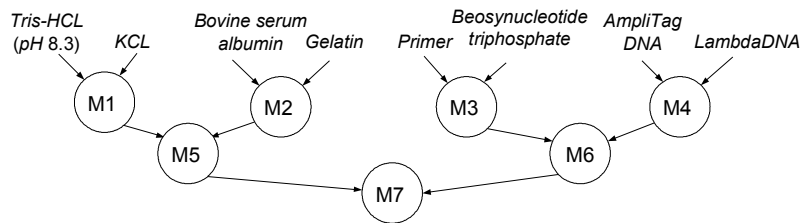

Figure 7: Sequencing graph for the mixing stage of PCR.

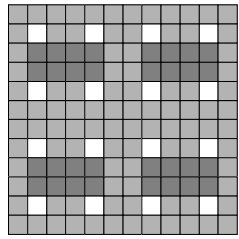

\begin{tabular}{|c|c|}
\hline $\begin{array}{c}\text { Tile } \\
1\end{array}$ & $\begin{array}{c}\text { Tile } \\
2\end{array}$ \\
\hline $\begin{array}{c}\text { Tile } \\
3\end{array}$ & $\begin{array}{c}\text { Tile } \\
4\end{array}$ \\
\hline
\end{tabular}

(a)

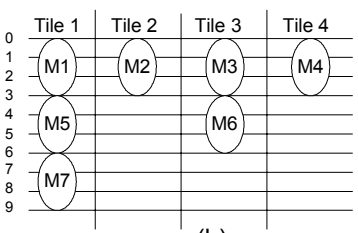

(b)
Figure 8: (a) A tile-based microfluidic biochip used in PCR. (b) Schedule and resource binding.

operation times associated with the mixers are obtained from real-life experiments [22]. The viscosities of the different samples in PCR are almost the same because they are highly diluted by the same fluid, such as $\mathrm{H}_{2} \mathrm{O}$, before dispensing. Thus, the times required for complete mixing of different droplets are equal for the same mixer. Based on the data available in [22] and the schedule shown in Figure 8(b), we determine the bioassay completion time for a fault-free array to be 9 seconds.

To evaluate the proposed defect tolerance schemes, the clustered defect model described in Section 4 is applied to this microfluidic biochip. Three different approaches, i.e., simple reconfiguration without graceful degradation, partial reconfiguration, and global reconfiguration, are evaluated through Monte-Carlo analysis (10000 simulation runs) respectively. Table 3 lists the values of $\mathrm{Y}$ and DEGR, as well as the CPU times (on a $1.0 \mathrm{GHz}$ Pentium-III PC with $256 \mathrm{MB}$ of RAM) for different values of the survival probability $p$. The results show that graceful degradation leads to a significant higher yield, but with a small increase in processing time. As expected, global reconfiguration outperforms partial reconfiguration in terms of yield and degradation level, but it requires more computation time.

Table 3: Results for PCR.

\begin{tabular}{|l|l|l|l|l|l|l|l|l|}
\hline \multirow{2}{*}{$\boldsymbol{p}$} & \multicolumn{3}{|l|}{ Baseline } & \multicolumn{3}{l|}{$\begin{array}{l}\text { Partial } \\
\text { Reconfiguration }\end{array}$} & \multicolumn{2}{l|}{$\begin{array}{l}\text { Global } \\
\text { Reconfiguration }\end{array}$} \\
\cline { 2 - 9 } & $\mathrm{Y}$ & $\begin{array}{l}\text { CPU } \\
\text { time(s) }\end{array}$ & $\mathrm{Y}$ & DEGR & $\begin{array}{l}\text { CPU } \\
\text { time(s) }\end{array}$ & Y & DEGR & $\begin{array}{l}\text { CPU } \\
\text { time(s) }\end{array}$ \\
\hline 0.1 & 0.0210 & 1.90 & 0.3318 & $104.2 \%$ & 2.39 & 0.9956 & $70.1 \%$ & 9.13 \\
\hline 0.2 & 0.0400 & 1.84 & 0.3793 & $91.6 \%$ & 2.41 & 0.9973 & $57.1 \%$ & 9.09 \\
\hline 0.3 & 0.0660 & 1.77 & 0.4352 & $83.3 \%$ & 2.27 & 0.9989 & $46.5 \%$ & 9.03 \\
\hline 0.4 & 0.1178 & 1.71 & 0.5040 & $71.6 \%$ & 2.31 & 0.9994 & $39.4 \%$ & 9.02 \\
\hline 0.5 & 0.1852 & 1.67 & 0.5625 & $60.4 \%$ & 2.23 & 0.9995 & $31.8 \%$ & 9.00 \\
\hline 0.6 & 0.2670 & 1.64 & 0.6392 & $49.7 \%$ & 2.29 & 1.0 & $25.2 \%$ & 8.92 \\
\hline 0.7 & 0.3885 & 1.58 & 0.7094 & $36.7 \%$ & 2.18 & 1.0 & $19.5 \%$ & 9.06 \\
\hline 0.8 & 0.5494 & 1.53 & 0.8046 & $25.0 \%$ & 2.12 & 1.0 & $13.9 \%$ & 8.88 \\
\hline 0.9 & 0.7507 & 1.49 & 0.8979 & $12.3 \%$ & 2.12 & 1.0 & $7.3 \%$ & 8.84 \\
\hline
\end{tabular}

6.2 Evaluation example 2: in-vitro diagnostics on human physiological fluids

We next evaluate the defect tolerance schemes using a set of concurrent bioassays. A behavioral description of multiplexed in-vitro diagnostics is as follows. Four types of human physiological fluids-plasma, serum, urine and saliva-are sampled and dispensed into the microfluidic biochip. Next each type of fluid is assayed for glucose, lactate, pyruvate and glutamate measurement. For each enzymatic assay, the droplets containing the suitably modified reagents (e.g., glucose oxidase, peroxidase, 4-AAP and TOPS for glucose measurement) are dispensed into the microfluidic array from the appropriate reservoirs. The result of the bioassays can be detected using 
optical absorbance measurement devices [4]. A sequencing graph modeling this set of bioassays is shown in Figure 9, where $M_{i}$ denotes the mixing operation and $D_{i}$ denotes the optical detection operation.

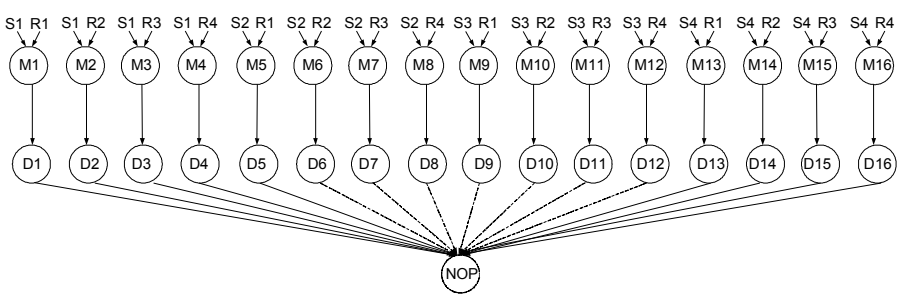

Figure 9: Sequencing graph for multiplexed clinical diagnostics.

We use a tile-based microfluidic biochip with $4 \times 4$ tiles for this set of bioassays. The corresponding operation schedule and resource binding are shown in Figure 10. Based on the data available in [18] and the schedule shown in Fig 10, the completion time for multiplexed diagnostics is 29 seconds.

As in the case of PCR, three defect tolerance schemes are evaluated using the clustered defect model. The mixing times for different human physiological fluids are different in various droplet mixers $[4,22]$. Table 4 lists the yields and degradation levels as well as CPU times for variant defect tolerance schemes. The results show that for a large set of bioassays as considered here, global reconfiguration offers better performance than partial reconfiguration. When the survival probability of a single tile is less than 0.8 , partial reconfiguration results in low yield (less than 50\%), but the yield for global reconfiguration approaches $100 \%$.

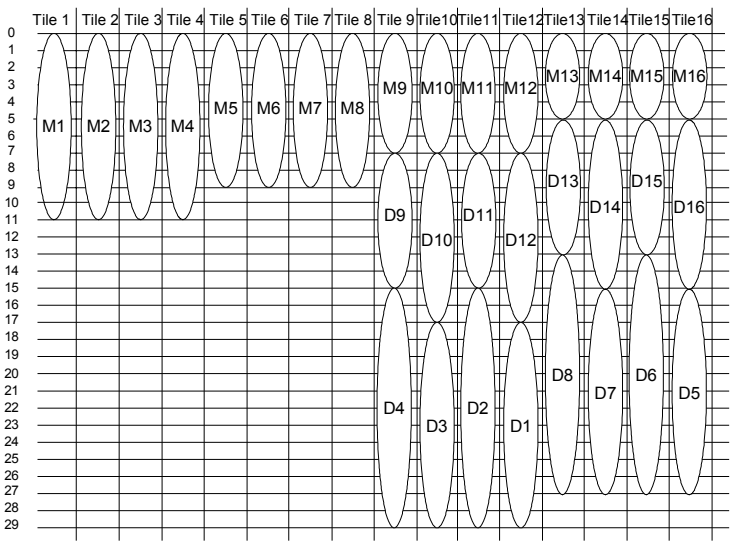

Figure 10: Schedule and resource binding.

Table 4: Results for multiplexed in-vitro diagnostics.

\begin{tabular}{|l|l|l|l|l|l|l|l|l|}
\hline \multirow{2}{*}{$\boldsymbol{p}$} & \multicolumn{2}{|l|}{ Baseline } & \multicolumn{3}{l|}{ Partial Reconfiguration } & \multicolumn{2}{l|}{$\begin{array}{l}\text { Global } \\
\text { Reconfiguration }\end{array}$} \\
\cline { 2 - 9 } & $\mathrm{Y}$ & $\begin{array}{l}\text { CPU } \\
\text { time(s) }\end{array}$ & $\mathrm{Y}$ & DEGR & $\begin{array}{l}\text { CPU } \\
\text { time(s) }\end{array}$ & $\mathrm{Y}$ & DEGR & $\begin{array}{l}\text { CPU } \\
\text { time(s) }\end{array}$ \\
\hline 0.1 & 0 & 6.20 & 0.0112 & $51.0 \%$ & 7.66 & 1.0 & $41.1 \%$ & 120.2 \\
\hline 0.2 & 0 & 5.99 & 0.0215 & $48.6 \%$ & 7.26 & 1.0 & $37.2 \%$ & 131.9 \\
\hline 0.3 & 0 & 5.71 & 0.0385 & $45.6 \%$ & 6.93 & 1.0 & $33.8 \%$ & 123.9 \\
\hline 0.4 & 0.0003 & 5.52 & 0.0627 & $43.5 \%$ & 6.64 & 1.0 & $30.7 \%$ & 130.0 \\
\hline 0.5 & 0.0017 & 5.24 & 0.1037 & $40.3 \%$ & 6.33 & 1.0 & $27.3 \%$ & 130.6 \\
\hline 0.6 & 0.0045 & 5.02 & 0.1653 & $35.8 \%$ & 6.21 & 1.0 & $23.6 \%$ & 135.3 \\
\hline 0.7 & 0.0249 & 4.76 & 0.2659 & $29.9 \%$ & 6.08 & 1.0 & $19.6 \%$ & 145.4 \\
\hline 0.8 & 0.0915 & 4.51 & 0.4106 & $22.9 \%$ & 6.20 & 1.0 & $14.7 \%$ & 142.4 \\
\hline 0.9 & 0.3127 & 4.23 & 0.6508 & $12.5 \%$ & 6.38 & 1.0 & $9.7 \%$ & 129.4 \\
\hline
\end{tabular}

\section{Conclusions}

We have presented a defect tolerance methodology for digital microfluidics-based biochips. This approach relies on a tile-based architecture and a graceful degradation method that retains full or partial functionality of a faulty tile. Two reconfiguration schemes, i.e., partial reconfiguration and global reconfiguration, have been developed to ensure that the bioassays mapped to the microfluidic array can be executed on the defective biochip through operation rescheduling and/or resource rebinding. Two real-life bioassays-PCR and a clinical diagnostic procedure-have been used to evaluate the proposed methodology. While partial reconfiguration is computationally more efficient, global reconfiguration leads to significant higher yield and a lower degradation level. The proposed defect tolerance methodology is expected to enhance yield and ensure high dependability of biochips targeted towards safety-critical applications. In our ongoing work, we are investigating a physical design methodology for biochips that incorporates defect tolerance in the design process.

\section{References}

[1] E. Verpoorte and N. F. De Rooij, "Microfluidics meets MEMS", Proceedings of the IEEE, vol. 91, pp. 930-953, 2003.

[2] W. Menz and A. Guber, "Microstructure technologies and their potential in medical applications", Minimally Invasive Neurosurgury, vol. 37, pp. 21-27, 1994.

[3] M. Pollack et al., "Electrowetting-based actuation of droplets for integrated microfluidics", Lab on a Chip, vol. 2, pp. 96-101, 2002.

[4] V. Srinivasan et al., "Clinical diagnostics on human whole blood, plasma, serum, urine, saliva, sweat, and tears on a digital microfluidic platform", Proc. $\mu$ TAS, pp. 1287-1290, 2003.

[5] International Technology Roadmap for Semiconductor (ITRS) http://public.itrs.net/Files/2003ITRS/Home2003.htm.

[6] S. Director et al., VLSI Design for Manufacturing: Yield Enhancement, Kluwer Academic Publishers, Norwell, MA, 1990.

[7] I. Koren and Z. Koren, "Defect tolerance in VLSI circuits: techniques and yield analysis", Proceedings of the IEEE, vol. 86, pp. 1819-1836, 1998.

[8] N. Howard et al., "The yield enhancement of Field-Programmable Gate Arrays", IEEE Trans. VLSI Systems, vol. 2, pp. 115-123, 1994.

[9] S. Kuo and I. Chen, "Efficient reconfiguration algorithms for degradable VLSI/WSI arrays", IEEE Trans. Computer-Aided Design, vol. 11, pp. 1289-1300, 1992.

[10] A. Kolpekwar and R. D. Blanton, "Development of a MEMS testing methodology", Proc. IEEE Int. Test Conf., pp. 923-93, 1997.

[11] N. Deb and R. D. Blanton, "Analysis of failure sources in surfacemicromachined MEMS", Proc. IEEE Int. Test Conf., pp. 739-749, 2000.

[12] S. Mir, B. Charlot and B. Courtois. "Extending fault-based testing to microelectromechanical systems." Journal of Electronic Testing : Theory and Applications, vol. 16, pp. 279-288, 2000.

[13] S. K. Tewksbury, "Challenges facing practical DFT for MEMS", Proc. Defect and Tolerance in VLSI Systems, pp. 11-17, 2001.

[14] H. G. Kerkhoff and H. P. A. Hendriks, "Fault modeling and fault simulation in mixed micro-fluidic microelectronic Systems", Journal of Electronic Testing: Theory and Applications, vol. 17, pp. 427-437, 2001.

[15] H. G. Kerkhoff and M. Acar, "Testable design and testing of microelectro-fluidic arrays", Proc. IEEE VLSI Test Sym., pp. 403-409, 2003.

[16] F. Su et al., "Testing of droplet-based microelectrofluidic systems", Proc. IEEE Int. Test Conf., pp. 1192-1200, 2003.

[17] F. Su et al, "Test planning and test resource optimization for dropletbased microfluidic systems”, Proc. European Test Sym., pp. 72-77, 2004.

[18] F. Su and K. Chakrabarty, "Architectural-level synthesis of digital microfluidics-based biochips", Proc. Int. Conf. Computer-Aided Design pp. 223-228, 2004

[19] M. G. Pollack, "Electrowetting-Based Microactuation of Droplets for Digital Microfluidics", PhD thesis, Duke University, 2001.

[20] A. D. Singh and C. M. Krishna, "On the effect of defect clustering on test transparency and IC test optimization", IEEE Trans. Computers, vol. 45, pp. 753-757, 1996.

[21] D. Lakin and A. D. Singh, "Exploiting defect clustering to screen bare die for infant mortality failures: an experimental study", Proc. IEEE Int Test Conf., pp. 23-30, 1999.

[22] P. Paik et al., "Rapid droplet mixers for digital microfluidic systems", Lab on a Chip, vol. 3, pp. 253-259, 2003.

[23] M. G. Pollack et al., "Investigation of electrowetting-based microfluidics for real-time PCR applications", Proc. $\mu$ TAS, pp. 619-622, 2003.

[24] T. Zhang et al, "Behavioral modeling and performance evaluation of microelectrofluidics-based PCR systems using SystemC", IEEE Trans. $C A D$, vol. 23, pp. 843-858, 2004. 